\title{
ESTUDIO DE LA TRACCIÓN VITREORRETINIANA PERIFÉRICA MEDIANTE HRA-II CON LENTE DE STAURENGHI 230
}

\section{PERIPHERAL VITREORETINAL TRACTION EXPLORATION BY STAURENGHI 230 LENS AND HRA-II}

\author{
LARA-MEDINA J ${ }^{1}$, ISPA-CALLÉN MC ${ }^{1}$, GONZÁLEZ-DEL-VALLE F ${ }^{1}$, NÚÑNEZ A ${ }^{1}$, \\ DOMÍNGUEZ MJ ${ }^{1}$, ARIAS-PALOMERO A ${ }^{1}$
}

\begin{abstract}
RESUMEN
Objetivo: Comunicar una nueva técnica de exploración de la retina periférica en pacientes con desprendimiento agudo de vítreo posterior.

Material y métodos: En una muestra de 65 pacientes diagnosticados de desprendimiento agudo de vítreo posterior se estudia la retina periférica mediante el HRA-II combinado con la lente panfunduscópica de Staurenghi SLO 230. El objetivo de esta técnica es la detección y localización de desgarros retinianos y de tracciones vítreo-retinianas.

Resultados: En nuestro estudio, utilizando la oftalmoscopía indirecta con indentación escleral encontramos en 11 pacientes al menos un desgarro retiniano. El HRA-II combinado con la lente de campo amplio fue capaz de detectar el $100 \%$ de dichas lesiones. Además, mediante esta técnica, se pudo localizar en 6 pacientes áreas de tracción vítreo retiniana, mostradas como zonas oscuras en la imagen infrarroja de fondo de ojo. Ninguno de los 65 pacientes refirió dolor ni fotofobia durante la exploración con esta lente de contacto. Los desgarros retinianos y las áreas de tracción fueron tratados con fotocoagulación con láser argón creando una
\end{abstract}

\begin{abstract}
Purpose: To report a new technique to explore peripheral vitreous-retinal tractions in patients with acute posterior vitreous detachment.

Material and methods: A population of 65 patients with acute posterior vitreous detachment was studied by panfunduscopic SLO 230 lens and HRA-II to detect peripheral retinal tears and vitreous-retinal tractions.

Results: In our study, using indirect ophthalmoscopic exploration, we identified eleven patients with at least one retinal tear. HRA-II combined with the Staurenghi SLO 230 lens was capable of detecting $100 \%$ of these lesions. In addition, by means of this technology, we identified dark retinal zones that corresponded to peripheral retinal traction in six patients. None of the 65 patients complained of pain or photophobia during the exploration with this contact lens. Retinal tears and peripheral vitreous-retinal traction were treated by argon laser photocoagulation to create a firm chorioretinal adhesion. In two patients we found new retinal tears in the follow-up, with these new lesions detected by indirect ophthalmoscopy and HRA-II with SLO 230 lens.
\end{abstract}

\footnotetext{
Recibido: 24/2/08. Aceptado: 14/12/09.

Servicio de Oftalmología. Complejo Hospitalario La Mancha-Centro. Alcázar de San Juan. Ciudad Real. España.

${ }^{1}$ Licenciado en Medicina.

Los autores manifiestan que no tienen interés comercial ni han recibido apoyo económico para la realización del presente trabajo.

Correspondencia:

Francisco Javier Lara Medina

Complejo Hospitalario La Mancha-Centro

Avenida de la Constitución

13600 Alcázar de San Juan (Ciudad Real)

España

E-mail: flarmed@hotmail.com
} 
adhesión coriorretiniana. En 2 pacientes se encontraron nuevos desgarros retinianos durante el seguimiento, siendo detectados tanto por oftalmoscopia indirecta como por el HRA-II con la lente panfunduscópica.

Conclusión: El HRA-II con la lente de campo amplio SLO 230 puede complementar la exploración de pacientes con desprendimiento agudo de vítreo posterior y ayudar a la detección de zonas traccionadas de la retina que podrían dar lugar a un desgarro retiniano.

Palabras clave: HRA-II, SLO, lente de Staurenghi, retina periférica, desgarro retiniano, desprendimiento de vítreo posterior, desprendimiento de retina.
Conclusion: HRA-II with the panfunduscopic Staurenghi SLO 230 lens can complement the exploration of patients with acute posterior vitreous detachment and can help to detect tractional zones that could develop into retinal tears (Arch Soc Esp Oftalmol 2009; 84: 625-630).

Key words: HRA-II, SLO, Staurenghi's lens, peripheral retina, retinal tear, posterior vitreous detachment, retinal detachment.

\section{INTRODUCCIÓN}

La incidencia anual de desprendimiento de retina regmatógeno (DRR) oscila aproximadamente entre 10 a 15 casos por cada 100.000 habitantes $(1,2)$. De ellos, un $20 \%$ han sido operados de cirugía de cataratas y un $10 \%$ son consecuencia de un traumatismo ocular (3-5).

Eventos precursores para el desarrollo de un DRR son el desprendimiento del vítreo posterior (DVP), desgarros retinianos sintomáticos, desgarros retinianos asintomáticos y las degeneraciones en empalizada. Aproximadamente el 15\% de los pacientes con desprendimiento de vítreo posterior presentan un desgarro retiniano en la exploración inicial (6). En aquellos pacientes con DVP sin desgarro retiniano inicial, existe un riesgo del 2 al 5\% de desarrollarlo en los días posteriores al inicio del cuadro. Un 50\% de los pacientes con un desgarro retiniano sintomático no tratado desarrollará un desprendimiento de retina regmatógeno $(7,8)$. Este riesgo se reduce a un 5\% si el desgarro retiniano es tratado en sus fases iniciales (9).

En la exploración de pacientes con sospecha de lesiones regmatógenas es fundamental el examen de la retina periférica. No hay síntomas que permitan distinguir un DVP con desgarro retiniano de un DVP sin desgarro, por lo que dicha exploración es obligatoria (10). El método de elección para la evaluación de la patología vitreorretiniana periférica es la oftalmoscopía indirecta combinada con depresión escleral, que puede complementarse mediante el uso de lentes de contacto con espejos. Si es imposible efectuar la evaluación de la retina periférica, está indicado la realización de una ecografía modob para descartar desgarros retinianos o un desprendimiento de retina, así como para buscar otras causas de hemorragia vítrea (10).

La oftalmoscopía mediante láser confocal (SLO) permite obtener imágenes dinámicas en alta resolución de la retina. Mediante un sistema de lentes de contacto podemos incrementar el campo convencional de exploración del SLO de $30-55^{\circ}$ hasta $150^{\circ}$, permitiendo una evaluación dinámica de la retina periférica.

Estudiamos la posibilidad de utilizar dicho angiógrafo en combinación con la lente panfunduscópica para evaluar la retina periférica en busca de zonas de tracción que sean susceptibles de ser tratadas para evitar un posible desgarro retiniano y posterior desprendimiento de retina.

\section{SUJETOS, MATERIAL Y MÉTODOS}

Para valorar la viabilidad de esta técnica, realizamos un estudio en pacientes diagnosticados de desprendimiento de vítreo posterior agudo sin desprendimiento de retina. Los pacientes son obtenidos del servicio de urgencias oftalmológicas del Hospital General La Mancha Centro (Alcázar de San Juan), a los cuales se les realiza una exploración oftalmológica completa, que incluye agudeza visual, presión intraocular, biomicroscopía de polo anterior y estudio de la retina central y periférica mediante oftalmoscopía indirecta combinada con indentación escleral. Finalmente, se procede a efectuar una exploración panfunduscópica con láser infrarrojo. 
Utilizamos un angiógrafo retiniano Heidelberg (HRA-II; Heidelberg Engineering GMBH, Dossenheim, Germany) con un láser de $835 \mathrm{~nm}$ de longitud de onda para obtener imágenes infrarrojas de la retina periférica, junto con una lente de contacto de campo amplio (Ocular Staurenghi 230 SLO Retina Lens; Ocular Instruments Inc, Bellevue, Wash). Esta lente de campo amplio está formada por dos lentes esféricas biconvexas y por dos elementos de contacto convexos-cóncavos. Tiene una magnificación de 0,23 y es afocal cuando se utiliza con gel gonioscópico. Todos los pacientes son estudiados bajo midriasis farmacológica, y tras la instilación de una gota de anestésico tópico, la lente de campo amplio se coloca en contacto con la córnea empleando gel gonioscópico. Empleando un láser de longitud de onda infrarroja se explora la totalidad de la retina periférica de dichos pacientes, ya que el movimiento del cabezal del angiógrafo permite acceder a zonas muy periféricas de la retina. Las áreas donde existe tracción vítreo-retiniana o bien está la retina en un plano diferente al resto (como ocurre en los desgarros retinianos) son visualizadas como oscuras, mientras que el resto de la retina sana adquiere un tono gris claro.

\section{RESULTADOS}

Estudiamos mediante el HRA-II y la lente de campo amplio a 65 pacientes diagnosticados de DVP agudo. Las características demográficas de dichos pacientes aparecen reflejadas en la tabla I. En todos los pacientes pudo visualizarse el gel vítreo móvil y desprendido flotando en la cavidad vítrea mediante la exploración dinámica con la lente de campo amplio. De los sesenta y cinco pacientes, 11 presentaban al menos un desgarro retiniano visualizado tanto por oftalmoscopia indirecta bajo indentación como por medio de la exploración infrarroja con la lente de campo amplio (figs. 1 y 2). Además, mediante esta última técnica se pudo observar en 6 pacientes áreas oscuras que corres-

Tabla I. Datos demográficos

Edad media

Mujeres

Hombres

Refracción media

Fáquicos

Pseudofáquicos

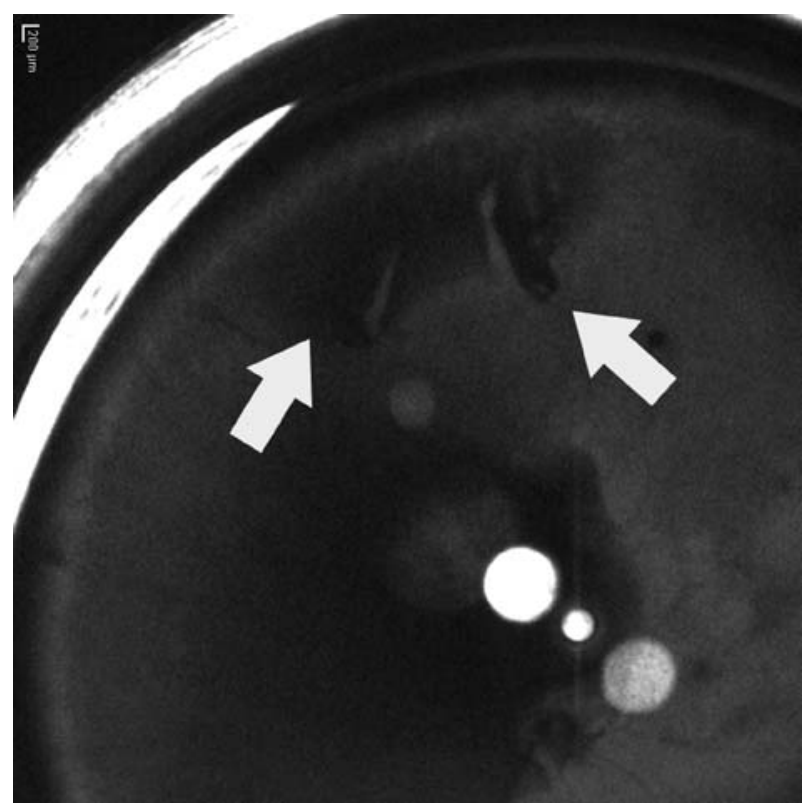

Fig. 1: El HRA-II permite identificar dos desgarros retinianos periféricos con desprendimiento de retina asociado (flechas blancas), en paciente con DVP hemorrágico (cabeza de flecha).

pondían a zonas retinianas traccionadas por mechas vítreas, como se pudo comprobar con una exploración posterior con lente de Goldmann de tres espejos (fig. 3). Ninguno de los 65 pacientes refirió dolor a la exploración con la lente de campo amplio ni fotofobia durante la realización de la prueba. Los

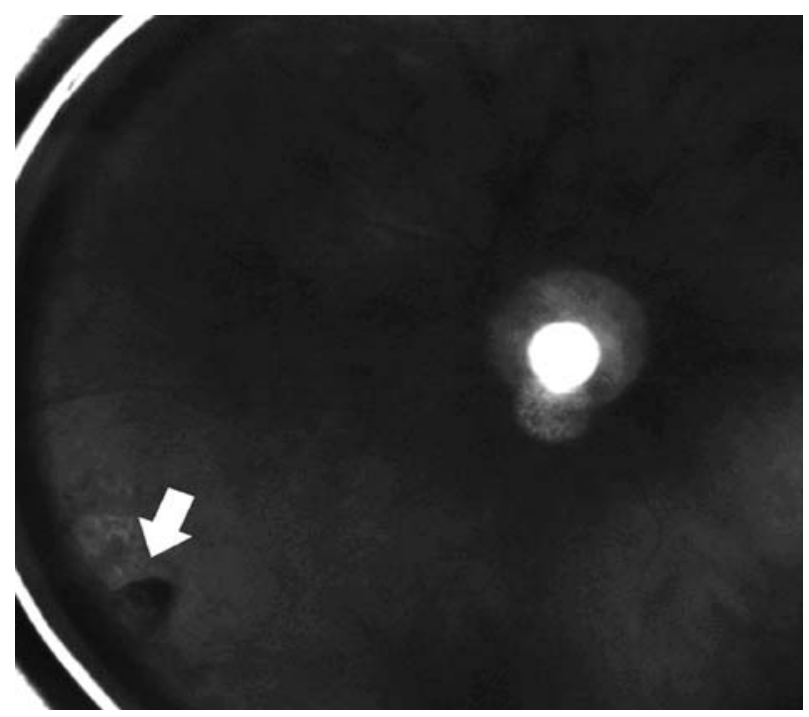

Fig. 2: Desgarro temporal superior (flecha blanca) en paciente pseudofáquico estudiado con la lente SLO 230. 


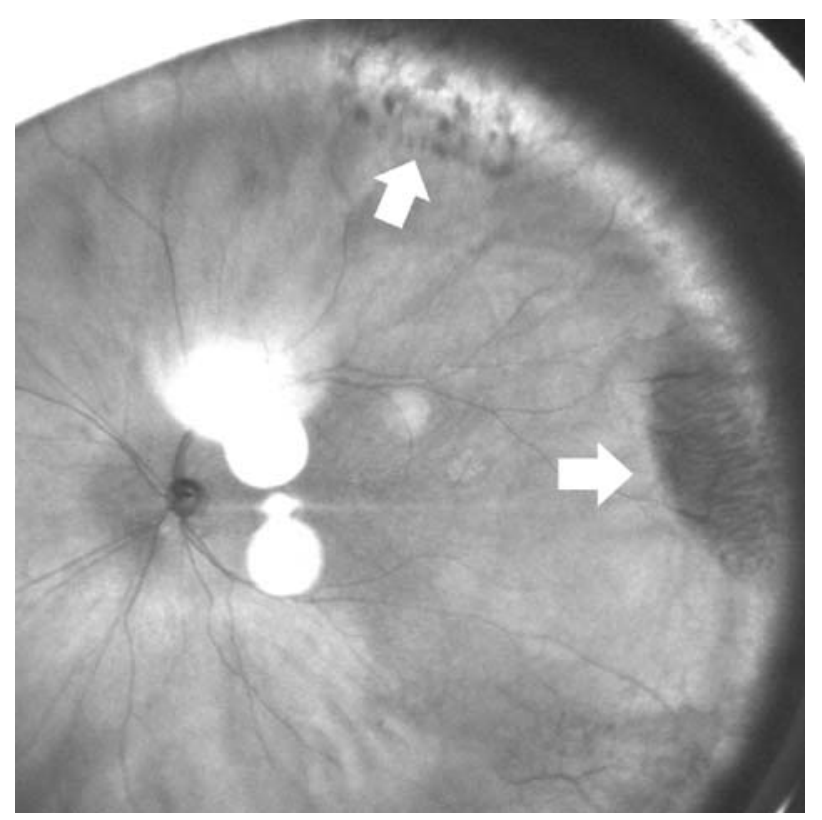

Fig. 3: El HRA-2 muestra dos áreas de tracción vitreoretiniana periférica (flechas blancas), correspondientes a zonas de blanco sin presión en la exploración oftalmoscópica.

pacientes en los que se encontró un desgarro retiniano o bien una zona de tracción vitreorretiniana fueron tratados mediante láser argón, con el fin de crear una adhesión coriorretinana que evitase la progresión a desprendimiento de retina. Todos los pacientes que no presentaban ninguna alteración salvo el DVP agudo fueron reexplorados al mes del episodio inicial, no encontrándose en ninguno de ellos nuevas lesiones regmatógenas. Los pacientes que recibieron láser argón se revisaron a las 2 semanas, hallándose en 2 de ellos nuevos desgarros retinianos, los cuales fueron tratados (fig. 4).

\section{DISCUSIÓN}

El diagnóstico precoz y el posterior tratamiento de los desgarros retinianos agudos reduce la tasa de pacientes que evolucionan a un desprendimiento de retina regmatógeno (9). Sin embargo, en algunas ocasiones, se puede generar un DRR a pesar del tratamiento apropiado con láser o crioterapia. Hasta en un $10-16 \%$ de estos casos tratados se desarrollan desgarros retinianos secundarios en zonas de tracción vítreo-retiniana (9). Esto es especialmente importante para pacientes pseudofáquicos, donde

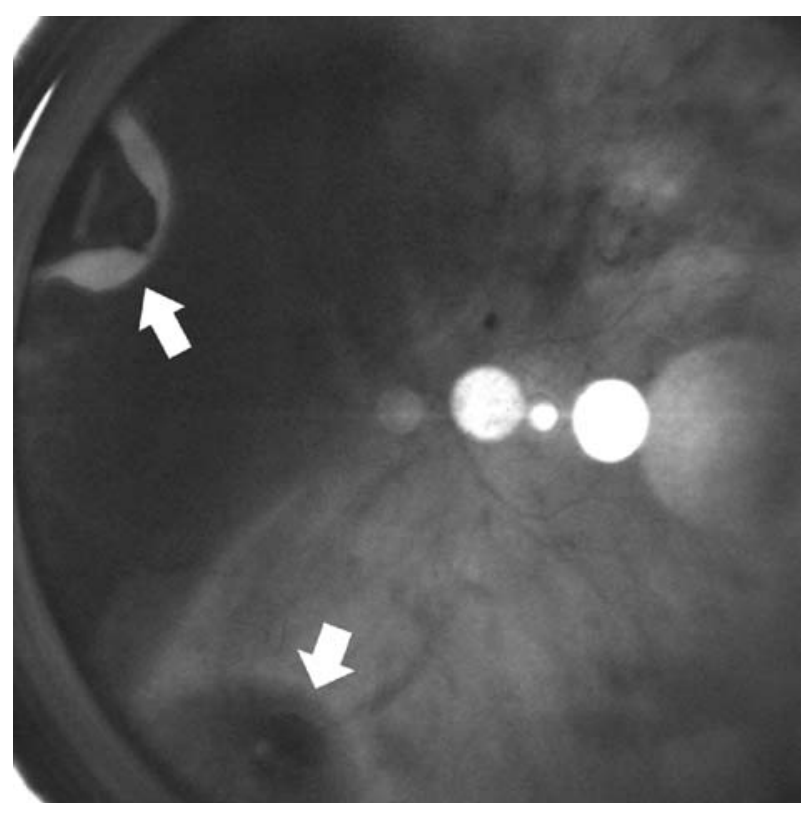

Fig. 4: Este paciente sufrió un primer desgarro retinano en la zona nasal superior y posteriormente desarrolló un segundo desgarro inferonasal con desprendimiento de retina asociado (zona oscura alrededor del desgarro).

existe una mayor frecuencia de desarrollar nuevos desgarros retinianos y por tanto aplicar retratamientos (9). Por ello es fundamental realizar en estos pacientes una exploración completa de la retina periférica, detectando las zonas de tracción retinana. Es obligado en pacientes con desprendimiento agudo del vítreo posterior la exploración de la retina periférica con indentación escleral (10). Sin embargo, esta exploración con frecuencia es dolorosa para el paciente y podemos infravalorar estas zonas predisponentes. La exploración mediante SLO con lente panfunduscópica nos puede servir de ayuda en estos pacientes, siendo una exploración complementaria a la indentación escleral. En esta prueba se pueden visualizar las zonas de desgarro retiniano o de tracción vítrea de forma oscura, distinguiéndose claramente del tono gris claro del resto de la retina. El SLO se basa en la utilización de un haz de láser enfocado en un determinado plano retiniano. Pensamos que al estar situada la retina en una posición más anterior por la tracción vítrea se produce un efecto de desenfoque y se visualiza más oscura. De hecho, si cambiamos el enfoque a un plano más anterior desaparece el color oscuro y la lengüeta de retina desprendida de un desgarro adquiere el mismo color grisáceo que la retina aplicada. 
Esta prueba aporta, en nuestra opinión, dos ventajas a los pacientes diagnosticados de desprendimiento agudo de vítreo posterior. En primer lugar, confirmaría y complementaría los hallazgos de la oftalmoscopía indirecta con indentación escleral, detectando los desgarros retinianos y las posibles zonas de tracción. En segundo lugar, es una prueba obtenida en formato digital, pudiendo mostrar al paciente y a sus familiares las lesiones retinianas periféricas, incluso de forma dinámica, pudiendo almacenarlas posteriormente en soporte informático. De esta forma, el paciente puede entender tanto el proceso que le afecta como el tratamiento que va a recibir.

Actualmente además esta exploración, existen varios sistemas para la obtención de imágenes retinianas de campo amplio. Existe un sistema de campo amplio basado en tecnología SLO de dos colores, denominado Panoramic200 (Optos plc, Dunfermline, Escocia) con menor resolución que los sistemas angiográficos de SLO. Otros sistemas de campo amplio no SLO utilizan una lente oftalmoscópica de contacto combinada con iluminación transescleral para adultos (Panoret 1000; Medibell Ltd, Haifa, Israel) o con iluminación transpupilar para pacientes pediátricos (RetCam II; Massie Research laboratorios Inc, Dublín, Calif).

El HRA-II en combinación con la lente panfunduscópica Staurenghi 230 puede ser utilizado en adultos y niños, si bien la lente puede resultar demasiado grande para niños de corta edad. Es también útil en pacientes afáquicos y pseudofáquicos, y por utilizar un láser infrarrojo puede resultar especialmente adecuado en pacientes con moderadas opacidades de medios, como pueden ser casos de cataratas y hemorragias vítreas no muy densas.

En nuestro estudio mostramos cómo la utilización de un HRA-2 junto con un sistema de campo amplio (lente Staurenghi SLO 230) puede comple- mentar la exploración de pacientes con desprendimiento agudo de vítreo posterior y ayudar a la detección de zonas traccionadas que pueden ocasionar un desgarro retiniano.

\section{BIBLIOGRAFÍA}

1. Haimann MH, Burton TC, Brown CK. Epidemiology of retinal detachment. Arch Ophthalmol 1982; 100: 289-292.

2. Wilkes SR, Beard CM, Kurland LT, Robertson DM, O'Fallon WM. The incidence of retinal detachment in Rochester, Minnesota, 1970-1978. Am J Ophthalmol 1982; 94: 670-673.

3. Javitt JC, Tielsch JM, Canner JK, Kolb MM, Sommer A, Steinberg EP. National outcomes of cataract extraction. Increased risk of retinal complications associated with Nd:YAG laser capsulotomy. The Cataract Patient Outcomes Research Team. Ophthalmology 1992; 99: 14871498.

4. Tielsch JM, Legro MW, Cassard SD, Schein OD, Javitt JC, Singer AE, et al. Risk factors for retinal detachment after cataract surgery. A population-based case-control study. Ophthalmology 1996; 103: 1537-1545.

5. Rowe JA, Erie JC, Baratz KH, Hodge DO, Gray DT, Butterfield L, et al. Retinal detachment in Olmsted County, Minnesota, 1976 through 1995. Ophthalmology 1999; 106: 154-159.

6. Scott IU, Smiddy WE, Merikansky A, Feuer W. Vitreoretinal surgery outcomes. Impact on bilateral visual function. Ophthalmology 1997; 104: 1041-1048.

7. Dayan MR, Jayamanne DG, Andrews RM, Griffiths $P G$. Flashes and floaters as predictors of vitreoretinal pathology: is follow-up necessary for posterior vitreous detachment? Eye 1996; 10: 456-458.

8. Van Overdam KA, Bettink-Remeijer MW, Mulder PG, Van Meurs JC. Symptoms predictive for the later development of retinal breaks. Arch Ophthalmol 2001; 119: 1483-1486.

9. Smiddy WE, Flynn HW Jr, Nicholson DH, Clarkson JG, Gass JD, Olsen KR, et al. Results and complications in treated retinal breaks. Am J Ophthalmol 1991; 112: 623631.

10. American Academy of Ophthalmology. Preferred practice pattern: Posterior Vitreous Detachment, Retinal Breaks, and Lattice Degeneration 2003. Disponible en: www.aao.org 